\title{
Dynamical-system models of transport: chaos characteristics, the macroscopic limit, and irreversibility
}

\author{
Jürgen Vollmer ${ }^{\mathrm{a}, \mathrm{b}, *}$, Tamás Tél $^{\mathrm{c}}$, Wolfgang Breymann ${ }^{\mathrm{d}}$ \\ ${ }^{a}$ Fachbereich Physik, Universität Essen, D-45117 Essen, Germany \\ ${ }^{\mathrm{b}}$ Max Planck Institute for Polymer Research, Ackermannweg 10, D-55128 Mainz, Germany \\ ${ }^{c}$ Institute for Theoretical Physics, Eötvös University, P.O. Box 32, H-1518 Budapest, Hungary \\ ${ }^{\mathrm{d}}$ Institute of Physics, University of Basel, Klingelbergstr. 82, CH-4056 Basel, Switzerland
}

\begin{abstract}
The escape-rate formalism and the thermostating algorithm describe relaxation towards a decaying state with absorbing boundaries and a steady state of periodic systems, respectively. It has been shown that the key features of the transport properties of both approaches, if modeled by low-dimensional dynamical systems, can conveniently be described in the framework of multibaker maps. In the present paper we discuss in detail the steps required to reach a meaningful macroscopic limit. The limit involves a sequence of coarser and coarser descriptions (projections) until one reaches the level of irreversible macroscopic advection-diffusion equations. The influence of boundary conditions is studied in detail. Only a few of the chaos characteristics possess a meaningful macroscopic limit, but none of these is sufficient to determine the entropy production in a general non-equilibrium state.
\end{abstract}

(C) 2003 Elsevier B.V. All rights reserved.

PACS: 05.70.Ln; 05.45.+b; 05.20.-y; 51.10.+y

Keywords: Chaos; Transport equations; Multibaker maps; Macroscopic limit

\section{Introduction}

The connection between non-equilibrium statistical physics and the underlying chaotic dynamics has recently attracted great attention [1-45]. Central questions are how the microscopic reversible dynamics can appear as an irreversible process on the macroscopic level, and how the macroscopic transport coefficients (like, e.g. diffusion or drift coefficients) are related to microscopic characteristics of the underlying chaotic dynamics. Interestingly, these problems can even be discussed in the framework of chaotic dynamical systems with only a few degrees of freedom. Multibaker maps [1,4,8,30-45] turned out to be particularly suited for this purpose since they show all generic features of spatially-extended, low-dimensional, hyperbolic dynamical systems, and are amenable to analytical calculations. Depending on the choice of parameters and boundary conditions various approaches to describe transport can be addressed:

\footnotetext{
* Corresponding author. Present address: AG Komplexe Systeme, Fachbereich Physik, Philipps Universität, 35032 Marburg/Lahn, Germany. E-mail address: juergen.vollmer@physik.uni-marburg.de (J. Vollmer).
} 
- Thermostating algorithm. An external force is used to invoke currents, and a constraint force acting on the particles is introduced to avoid the growth of the kinetic energy without bound [2,5,6,11,16-18,22]. The constraint force simulates the presence of an internal thermostat. It preserves time-reversibility, but makes the particle dynamics dissipative on average. The systems are assumed to be periodic of large spatial extension, and the long-time dynamics exhibits sustained chaos on an underlying chaotic attractor. Once the dynamics collapsed to the attractor the transport coefficients can be connected with the average phase-space contraction rate [2,5,6,11,12].

- Escape-rate formalism. Open systems of large spatial extensions are considered [3,4,8,10,32-34]. In such cases the particle dynamics is chaotic in the sense of transient chaos, and there exists an underlying non-attracting chaotic set, a chaotic saddle in the phase space. The particle motion is a kind of scattering process, and the transport coefficients are related $[4,10,30]$ to the chaotic saddle's escape rate (hence the name escape-rate formalism).

An open system with fixed densities at the boundaries gives rise to a stationary flow of particles through the system. These systems lie, however, beyond the scope of dynamical-systems theory, and the associated fractal structures have been analyzed elsewhere in considerable detail $[4,25,26,31,35-37,41]$. Therefore, such systems will not further be discussed here.

We investigate a generalized multi-strip baker chain, and show how irreversibility arises in this system by applying coarse graining. It is illustrated how, via a sequence of coarser and coarser observations (namely: projection of the dynamics onto the transport direction, averaging over the motion inside cells, taking the limit of continuous time, and of large linear scales) one reaches the level of macroscopic equations. One of the merits of multibaker maps is that due to their straightforward chaotic dynamics these steps can explicitly be worked out-as opposed to the classical discussions of taking these limits in the 1960s [47-49] where the initial steps could only heuristically be addressed. The influence of boundary conditions is studied in detail and we come to the conclusion that their effects are important even in the large system limit.

The aim of the present paper is to show how far one can go in deriving macroscopic transport equations based on a low-dimensional, dynamical system (which, by definition, is restricted to a finite phase space) as underlying "microscopic" dynamics. Only models with periodic and with absorbing-boundary conditions correspond to dynamical systems. They possess a natural measure, and we will hence be able to address the question whether the chaos characteristics associated with this measure can play a role in the macroscopic description of the related transport processes. Most of the characteristics are ruled out by the observation that they are not well defined in the macroscopic limit in which the coarse-grained dynamics gives rise to macroscopic transport equations compatible with irreversible thermodynamics. The two major exceptions are the average phase-space contraction rate and the escape rate. We point our, however, that none of these is sufficient to describe the thermodynamic entropy production in a general macroscopically inhomogeneous state.

The paper is organized as follows. In Section 2 the multibaker chain is defined and the most important special cases are identified. In Section 3 we start from the microscopic chaotic dynamics of a long chain and go through a sequence of coarse-graining procedures to end up with the macroscopic advection-diffusion equation. Technical details of determining the characteristics of the microscopic dynamics are relegated to Appendix A. In Section 4 we discuss the effects of periodic and absorbing-boundary conditions used for the thermostating algorithm and the escape-rate formalism. Section 5 is devoted to a comparison of quantities with a well-defined macroscopic limit (decay and phase-space contraction rates) in the periodic and open cases. The paper is concluded by a discussion (Section 6).

\section{The multibaker chain}

The single-particle phase space of a multibaker model is a rectangle of size $[0, N a] \times[0, b]$. It comprises a chain of $N$ identical cells of linear size $a$ coupled to each other along the $x$-axis (Fig. 1a). Each cell possesses the same 


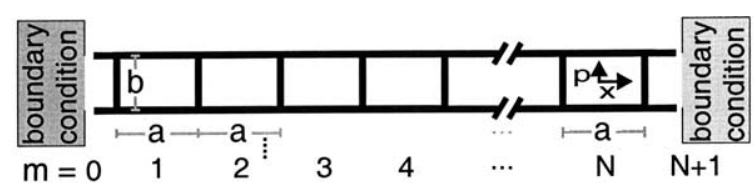

(a)

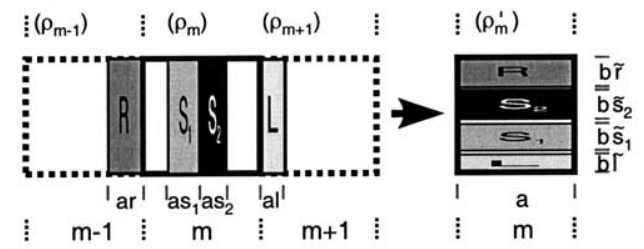

(b)

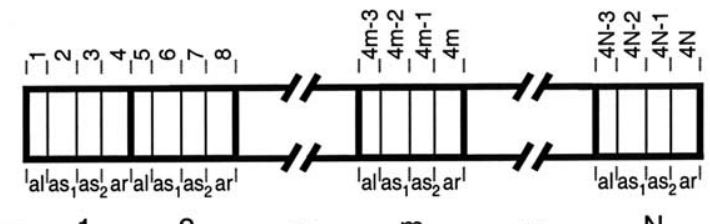

(c)

Fig. 1. The multibaker model (microscopic dynamics). (a) Geometry of the chain: every rectangle of size $a \times b$ contains a baker map with possible escape to and entering from its neighbors. The respective cells are labeled by the index $m$ running from 1 to $N$. Boundary conditions are implemented by appropriate choices of the dynamics in additional cells $m=0, N+1$ at the outer ends of the chain. (b) Action of the multibaker map on a single cell in the case of a four-element partition, $k=2$. Four vertical columns in cell $m-1, m$ and $m+1$, respectively, are squeezed and stretched by the map such that the resulting horizontal strips fit into cell $\mathrm{m}$. The height and width of the columns and strips are indicated on the margin. (c) Generating partition for $k=2$. The $4 N$ vertical strips of the chain form a generating partition for a symbolic dynamics describing the chaotic dynamics. The symbols are given atop of the columns.

internal dynamics, carried out at integer multiples of a discrete time unit $\tau$. This action is defined here in a pictorial manner in Fig. 1b. The total area $a b$ of the cell is divided into $k+2$ vertical columns of widths $l a, s_{1} a \cdots s_{k} a$ and $r a$ such that

$$
l+r+\sum_{i=1}^{k} s_{i}=1 .
$$

The presence of a bias is expressed by a difference in the areas mapped to the left $(l)$ and right $(r)$.

Fig. $1 \mathrm{~b}$ illustrates the action of the map for $k=2$. The branches mapped into cell $m$ are indicated by the labels $L, S_{1}, S_{2}$, and $R$, respectively. The branches $L$ and $R$ are mapped onto horizontal strips of width $a$ and of heights $\tilde{l} b$ and $\tilde{r} b$, respectively, in cells joint immediately to the right and left of the respective initial cells. The images of the middle vertical columns remain inside the original cell and are all stretched to horizontal strips of widths $a$ and height $\tilde{s}_{1} b \cdots \tilde{s}_{k} b$. The parameters $s_{i}, \tilde{s}_{i}$ specify the internal dynamics of the cells, while $l, \tilde{l}$ and $r, \tilde{r}$ characterize the coupling between neighboring cells. We are interested in cases where these horizontal strips fit into the neighboring cells without overlapping with the images of the columns not leaving the cell. Thus, the dynamics is injective on the chain of cells. The sum of the twiggled quantities can be smaller than unity,

$$
\tilde{l}+\tilde{r}+\sum_{i=1}^{k} \tilde{s}_{i} \leq 1,
$$

indicating the possibility of global phase-space contraction (the dynamics need not be surjective). 
For more than one strip staying inside a cell, $k>1$, the transient dynamics of a single cell with open ends is already chaotic. The full chain's dynamics is, however, chaotic for any $k$ (including $k=0,1$ ) due to the coupling of a large number of identical cells.

This multibaker map represents a rather broad class of dynamical systems, and we believe that the results not depending on its specific features will be of general validity for hyperbolic dynamical systems with bias and transport. Depending on the choice of the local phase-space contraction ratios (Jacobians)

$$
J_{\mathrm{L}}=\frac{\tilde{l}}{l}, \quad J_{\mathrm{R}}=\frac{\tilde{r}}{r}, \quad J_{i}=\frac{\tilde{s}_{i}}{s_{i}}, \quad i=1, \ldots, k,
$$

different classes of time-evolution equations can be modeled:

(a) Hamiltonian (i.e., area-preserving) dynamics:

$$
J_{\mathrm{L}}=J_{\mathrm{R}}=J_{i}=1, \quad i=1, \ldots, k,
$$

(b) homogeneous dissipation:

$$
J_{\mathrm{L}}=J_{\mathrm{R}}=J_{i} \equiv J<1, \quad i=1, \ldots, k,
$$

(c) thermostated dynamics:

$$
J_{\mathrm{L}}=\frac{1}{J_{\mathrm{R}}}=\frac{r}{l}, \quad J_{i}=1, \quad i=1, \ldots, k
$$

The last choice is a model of thermostating since it reflects the following basic features:

(i) The dynamics is area-contracting (expanding) if the trajectory moves in the direction of (against) the bias. This mimics the effect of a slowing down (acceleration) of particles moving parallel to (against) the external field $[2,3,5,8,34]$.

(ii) Volume elements that move away but finally come back to the original position pick up no net contribution to phase-space contraction, i.e., the thermostat only acts when work is done on the system.

(iii) The mapping of the phase space is one-to-one so that the stationary distribution is supported by the full phase space, on which the dynamics is ergodic.

\section{Dynamics on different levels of coarse graining}

\subsection{Full microscopic dynamics}

The basic dynamics on the phase space introduced in the previous section can be written as a map

$$
M:\left(x_{n}, y_{n}\right) \mapsto\left(x_{n+1}, y_{n+1}\right)
$$

acting at integer multiples of the microscopic time unit $\tau$, i.e., in continuous time at $t=n \tau$. The explicit form of $M$ is easy to find from its action shown in Fig. 1. From the point of view of statistical properties and transport, the time evolution of the densities is of central importance. Let $\varrho_{n}(x, y)$ denote a phase-space distribution at time $n \tau$. The Liouville operator $\mathcal{L}$ connects $\varrho_{n}$ with $\varrho_{n+1}$ :

$$
\mathcal{L}: \varrho_{n} \mapsto \varrho_{n+1}
$$


In the present system it can be written as a transfer matrix $T$. To construct the matrix we observe that due to the piecewise-linear character of the multibaker map a (piecewise) constant phase-space density $\varrho(x, y)$ remains piecewise constant under the time evolution. Moreover, any smooth initial condition converges to the same asymptotic distribution (which is irregularly changing in the $y$-direction, but is constant in each cell along the $x$-axis). Therefore, in what follows we restrict ourselves to piecewise constant initial conditions. In order to find the constant-density regions, a partitioning of the chain by the horizontal strips is considered, which are defined by the one-step backward dynamics in overlap with the vertical columns. There are then, respectively, $k+2$ strips and columns in each cell. In cell $m, m=1,2, \ldots, N$, the symbols $(k+2) m-(k-1),(k+2) m-(k-2), \ldots,(k+2) m$ mark the columns, and the strips are labeled by the same set of numbers running now from bottom to top (cf. Fig. 1c). This partition is generating and Markovian [51,53]. Consequently, it specifies a symbolic dynamics of $(k+2) N$ symbols. In such a situation the transfer matrix plays the role of the Liouville operator [52]. In the present setting it takes the form

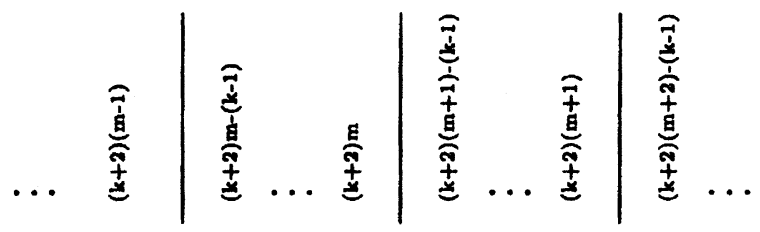

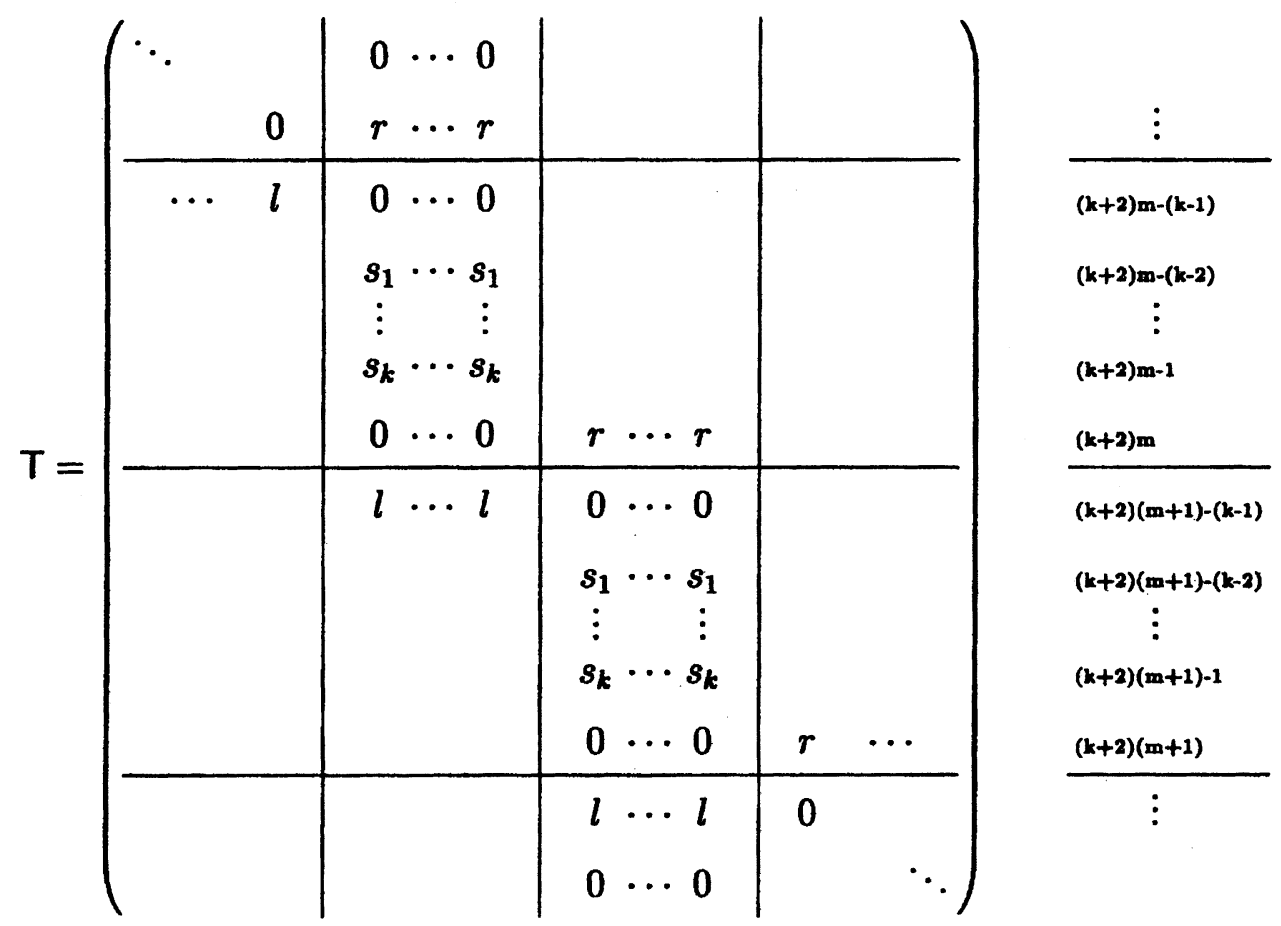

where-as indicated at the top and right of the matrix - the horizontal and vertical rules group together $k+2$ columns comprising a given cell (cell $m$ and $m+1$ are indicated). All elements that are not explicitly given in (3.2) vanish. The other elements $T_{\alpha, \beta}$ of T represent non-vanishing probabilities to be mapped from a column of code $\alpha$ into a column of code $\beta$, where $\alpha$ and $\beta$ label the rows and columns of $\mathrm{T}$, respectively. The time evolution of the piecewise constant phase-space density amounts to repeated application of this matrix on a vector representing the initial condition. Consequently, the long-time properties of the dynamics will be connected with the largest eigenvalues of the transfer matrix $\mathrm{T}$. 
Since the entries in every block of $k+2$ columns are identical every left eigenvector consists of blocks of $k+2$ identical components. Consequently, eigenvector elements do not vary within the $k+2$ entries characterizing a cell. This allows us to restrict our attention to transitions between neighboring cells only. A reduced transfer matrix $\overline{\mathrm{T}}$ of size $N \times N$ (or possibly $(N+2) \times(N+2)$ when additional cells are needed to implement the boundary conditions, cf. Section 4 and Appendix A) can be defined with the transition probabilities from a cell of index $m$ to another one as $\bar{T}_{m, m-1}=l, \bar{T}_{m, m}=\sum_{i=1}^{k} s_{i} \equiv s$, and $\bar{T}_{m, m+1}=r$. For the cells in the interior of the chain, all other transitions are forbidden. Consequently,

$$
\overline{\mathrm{T}} \equiv\left(\begin{array}{ccccccc}
\ddots & \ddots & \ddots & & & & \\
& l & s & r & & & \\
& & l & s & r & & \\
& & & l & s & r & \\
& & & & \ddots & \ddots & \ddots
\end{array}\right)
$$

is tridiagonal, up to entries in the outermost rows or columns, which depend on the choice of boundary conditions.

The spectrum of the reduced transfer matrix is worked out in Appendix A. It fully characterizes all decay rates of the forward dynamics. Moreover, the thermodynamic formalism of dynamical systems [53] implies that structurally identical matrices describe the whole set of multifractal properties of the microscopic dynamics. The various characteristics only differ by the choice of the non-vanishing matrix elements. Accordingly, in Appendix A the spectrum of tridiagonal matrices is worked out for arbitrary positive values for $r, s$ and $l$. We thus obtain a description of all relevant dynamical and geometrical (fractal) properties of the invariant sets including Lyapunov exponents, generalized dimensions along both the stable and the unstable directions, and Rényi entropies. Since different boundary conditions lead to different positions of the non-vanishing matrix elements in the outermost rows and columns, the exact form of the spectrum depends on the type of boundary conditions (cf. Eqs. (A.16) and (A.11)).

\subsection{Microscopic dynamics reduced to the direction of transport}

Having started with the full microscopic treatment of the multibaker dynamics, we now take a successively more macroscopic point of view of the description, and state compatibility conditions.

Due to the special form of the multibaker map, the density does not depend at any time on the $x$-coordinate inside a cell. In other words, the $y$-dependence is exactly the same for all $x$ values inside a cell. We can therefore easily integrate the phase-space density over the $y$-coordinate, obtaining the time evolution of the projected density (beware of the different symbols $\rho$ and $\varrho$ ):

$$
\rho_{n}(x)=\int_{0}^{b} \mathrm{~d} y \varrho_{n}(x, y) .
$$

As a consequence, the $x$ dynamics can be described by a one-dimensional map $f(x)$ depicted in Fig. 2 . Since the baker map is piecewise linear, $f(x)$ is of the same character. The time evolution of its reduced density is described by the Frobenius-Perron equation [51], which takes the general form

$$
\rho_{n+1}\left(x^{\prime}\right)=\sum_{x \in f^{-1}\left(x^{\prime}\right)} \frac{\rho_{n}(x)}{\left|f^{\prime}(x)\right|} .
$$




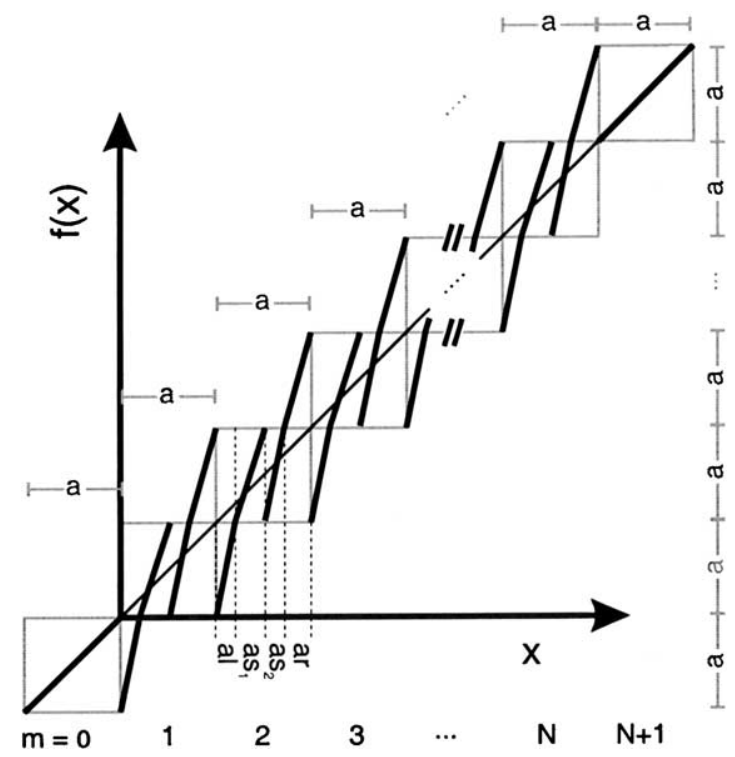

Fig. 2. The one-dimensional map $f(x)$ obtained by projecting the multibaker dynamics to the $x$-axis $(k=2)$.

In the present case every $x^{\prime}$ in $[0, N a]$ has $k+2$ preimages: $x_{\mathrm{L}}\left(x_{\mathrm{R}}\right)$ in the left (right) neighboring cell, and $x_{i}$, $i=1, \ldots, k$ inside the same cell as $x^{\prime}$. The corresponding slopes $f^{\prime}(x)$ are $r^{-1}\left(l^{-1}\right)$ and $s_{i}^{-1}$, respectively, such that the Frobenius-Perron equation takes the form

$$
\rho_{n+1}\left(x^{\prime}\right)=r \rho_{n}\left(x_{\mathrm{L}}\right)+\sum_{i=i}^{k} s_{i} \rho_{n}\left(x_{i}\right)+l \rho_{n}\left(x_{\mathrm{R}}\right) .
$$

It is worth noting that even if the original dynamics was invertible (like, for instance, in the Hamiltonian or thermostated cases), the time evolution of the probabilities is irreversible due to the projection (3.4) on the $x$-axis. In other words, the process becomes irreversible since a kind of coarse graining has been applied. Eq. (3.6) associates the projected dynamics with a dissipative, ${ }^{1}$ one-dimensional map which has a unique attracting stationary solution (possibly identically zero) in the space of the $\rho(x)$. Owing to the piecewise-linear character of the map and the stretching property out of the elementary interval, $\rho_{n}(x)$ is constant in each cell. ${ }^{2}$ Therefore, we can represent the distribution at time $n$ by a vector $\vec{\rho}_{n}$ of $N$ elements $\rho_{n ; m}, m=1, \ldots, N$, and observe that for every $x$ in cell $m$ the terms appearing in (3.6) take the form $r \rho_{n}\left(x_{\mathrm{L}}\right)=r \rho_{n ; m-1}, \sum s_{i} \rho_{n}\left(x_{i}\right)=s \rho_{n ; m}$, and $l \rho_{n}\left(x_{\mathrm{R}}\right)=l \rho_{n ; m+1}$. Consequently, the Frobenius-Perron equation reads

$$
\vec{\rho}_{n+1}=\mathrm{H} \vec{\rho}_{n}
$$

A comparison of (3.6) with the definition of the reduced transfer matrix (3.3) leads to the observation

$$
\mathrm{H}=\overline{\mathrm{T}}^{+} \text {. }
$$

\footnotetext{
${ }^{1}$ The phase-space contraction rate of (3.6) is formally infinite, as for any one-dimensional map. After all, the phase-space contraction rate diverges in the limit where a higher-dimensional map reduces to the one-dimensional dynamics.

${ }^{2}$ We utilize at this point that any smooth initial density along the multibaker chain converges exponentially fast (on the time scale of the reciprocal value of the average positive Lyapunov exponent) to a distribution which is constant in each cell. Disregarding these short transients, we assume from here on that the density is constant within each cell.
} 
The Frobenius-Perron operator $\mathrm{H}$ is the transpose of the reduced transfer matrix. Correspondingly, the left eigenvectors of $\overline{\mathrm{T}}$ are the right eigenvectors of $\mathrm{H}$, among which the one belonging to the largest eigenvalue provides the natural invariant density along the $x$-axis. It is worth noting that the application of the thermodynamic formalism leads again to tridiagonal transfer matrices with the same structure as $\mathrm{H}$ but with different non-vanishing elements. Therefore, on this level we are still in a position to recover dynamic characteristics of the motion along the $x$-axis, but we have already lost information about the properties along the $y$-axis. In particular, the correct phase-space contraction rate can only be obtained from the full microscopic dynamics.

\subsection{Inter-cell dynamics: the random-walk picture}

From the point of view of modeling transport, a restriction of the attention to the average density in each cell corresponds to the existence of a smallest resolution $a$ in the configuration space (here, the $x$-axis) below which no spatial structure is resolved, and to an averaging over all the momenta in the respective regions. A reduction of the dynamics in this spirit is unavoidable in order to obtain a thermodynamic description [46-50]. For the multibaker map this amounts to a projection of the (deterministic) dynamical system onto a random walk: move left, right or stay with given probabilities. This projection illustrates that a deterministic chaotic dynamics can lead to a fully stochastic behavior after appropriate coarse graining (this was the basic assumption underlying, e.g. [49]). Note that on this level of the description there are no partitions inside a cell any longer: the role of mixing of the microscopic dynamics is taken over by the stochastic character of the random walk.

Let $P_{n ; m}$ denote the probability to find a particle in cell $m$ at discrete time $n$. According to the theory of Markov chains [56], the conservation of probability requires that the net flux through the boundaries of each cell amounts to the temporal change of the cell probability:

$$
P_{n+1 ; m}=(1-l-r) P_{n ; m}+l P_{n ; m+1}+r P_{n ; m-1} .
$$

Thus, the evolution of the vector $\vec{P}_{n} \equiv\left(P_{n ; 1}, \ldots, P_{n ; N}\right)$ is governed by the matrix equation

$$
\vec{P}_{n+1}=\mathrm{A} \vec{P}_{n},
$$

where the matrix elements of $A$ are the transition probabilities between neighboring cells. Its non-vanishing entries are

$$
A_{m, m-1}=l, \quad A_{m, m}=1-l-r \equiv s, \quad A_{m, m+1}=r .
$$

Due to the simple structure of the multibaker map the dynamics of the Markov chain and the projected 1D map are described by the same transition matrix: $A=\bar{T}$. However, the Markov chain dynamics is defined only between cells, not inside a given cell.

\subsection{Continuous-time dynamics: the master equation}

On the next level of coarse graining we only consider slow "macroscopic" time evolutions with continuous time $t$ defined as $t=n \tau$. We expect that the continuous-time macroscopic behavior differs from the microscopic one, and inherits properties of the latter via transport coefficients only. Physically this implies a separation of time scales. For a meaningful limit, $P_{n ; m} \rightarrow P_{m}(t=n \tau)$, of the probability distribution the jumping probabilities are required to be of the order of the microscopic time unit $\tau$. This induces the staying probability $s=1-r-l$ to be of order unity. Writing

$$
l=\mu \tau, \quad r=\lambda \tau,
$$


where $\mu$ and $\lambda$ are independent of $\tau$, we recover the master equation of a birth and death process [56]

$$
\frac{\mathrm{d} P_{m}(t)}{\mathrm{d} t}=\lambda P_{m-1}(t)+\mu P_{m+1}(t)-(\lambda+\mu) P_{m}(t)
$$

with constant coefficients $\lambda$ and $\mu$. The matrix governing this continuous-time dynamics has the same structure as the reduced transition matrix $\overline{\mathrm{T}}$ of the microscopic Liouville operator. Therefore, its full spectrum can easily be obtained by the methods applied in Appendix A. The fact that according to (3.12) the jumping probabilities $r$ and $l$ scale linearly in $\tau$ assures that the time evolution on macroscopic scales is slow as compared to the time unit $\tau$.

\subsection{Large-scale dynamics: the advection-diffusion equation}

As a final step we consider the limit of large spatial extension realized by a chain of length $L \equiv(N+1) a \gg a$ composed of $N \gg 1$ cells. To this end we consider the space variable $x \equiv m a$ to be continuous, i.e., we only resolve spatial variations on scales $x \gg a$. In order to have a meaningful limit $P_{m}(t) \rightarrow P(x=m a, t)$ for $m \gg 1$, the sum of the jumping probabilities must be much larger than their difference. Writing

$$
\lambda+\mu=\frac{2 D}{a^{2}}, \quad \lambda-\mu=\frac{v}{a}
$$

with $D$ and $v$ constant, and assuming weak spatial gradients $\left|P_{m}-P_{m-1}\right| \ll P_{m}$, the Fokker-Planck (or advectiondiffusion) equation

$$
\frac{\partial P(x, t)}{\partial t}=-v \frac{\partial P(x, t)}{\partial x}+D \frac{\partial^{2} P(x, t)}{\partial x^{2}}
$$

is recovered from (3.13) [57]. In this picture $D$ and $v$ are interpreted as diffusion coefficient and drift (or bias), respectively. By this sequence of projections and limits, we thus have achieved an equation, which describes the macroscopic time evolution of the system. For this reason we call [34] the limit the macroscopic limit.

From (3.12) and (3.14) we find that the original jump probabilities scale as

$$
\begin{aligned}
& l=\mu \tau=\frac{D \tau}{a^{2}}\left(1-\frac{v a}{2 D}\right), \\
& r=\lambda \tau=\frac{D \tau}{a^{2}}\left(1+\frac{v a}{2 D}\right) .
\end{aligned}
$$

In order to be compatible with an advection-diffusion description $v$ and $D$ must not depend on the time and space units $\tau$ and $a$.

Although not related to the advection-diffusion equation, it is worth introducing the backward jumping rates $\tilde{r}$ and $\tilde{l}$ as

$$
\begin{aligned}
& \tilde{l}=J \frac{D \tau}{a^{2}}\left(1-\varepsilon \frac{v a}{2 D}\right), \\
& \tilde{r}=J \frac{D \tau}{a^{2}}\left(1+\varepsilon \frac{v a}{2 D}\right) .
\end{aligned}
$$

Here, $J$ denotes the global Jacobian $J \equiv(\tilde{r}+\tilde{l}) /(r+l)$ on the strips contributing to transport, and $\varepsilon$ is a parameter measuring the deviation from constant phase-space contraction. The form (3.17) of expressing the backward rates is convenient for taking the macroscopic limit of the phase-space contraction rate. The three basic dynamics defined in Section 2 are recovered by the choices: (a) $J=1, \varepsilon=1$ (Hamiltonian case), (b) $J<1, \varepsilon=1$ (homogeneous dissipation), and (c) $J=1, \varepsilon=-1$ (thermostating). 

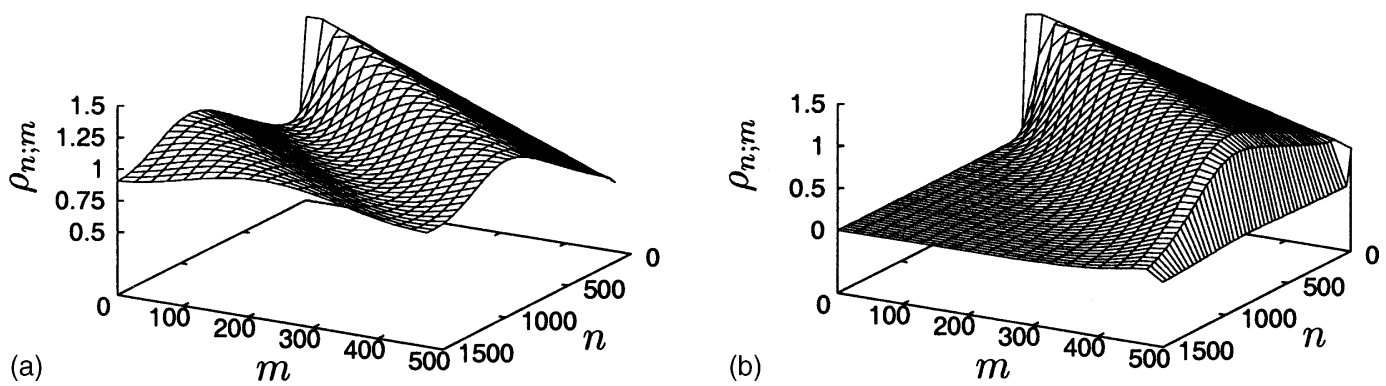

Fig. 3. Time evolution of $\rho_{n ; m}$ for $N=500, r=0.052$, and $l=0.048$. The initial density is linear, $\rho_{0 ; m}=(1.5-m / N)$. (a) Decay for periodic boundary conditions. The normalization $\sum_{m=1}^{N} \rho_{n ; m}=N$ of $\rho_{n ; m}$, and hence the average number of particles in the system does not change. (b) Decay for absorbing-boundary conditions. Asymptotically the overall density profile is constant in space and exponentially decaying in time. For $r>l$ a maximum appears close to the cells at the right boundary of the chain.

\section{Effects of boundary conditions}

The eigenvectors $\rho_{m}$ of the tridiagonal matrices $\overline{\mathbf{T}}^{+}$are exponential functions of subscript $m$, which in our case coincides with the cell index. The actual form of the eigenvectors and the eigenvalues depends on the boundary conditions defined by the action in boundary cells with indices $m=0$ and $m=N+1$. The two cases of periodic and absorbing-boundary conditions are considered in turn:

(i) Periodic boundary conditions: $\rho_{0}=\rho_{N}$, and $\rho_{N+1}=\rho_{1}$. For the transfer matrices this implies that, besides the tridiagonal structure described above, there are entries in the lower left and upper right corner chosen such that the entries of every line and of every column add up to $l+s+r$. In this case, the eigenvector represents a traveling wave (cf. Fig. 3a) of the form $\exp (\mathrm{i} 2 \pi \nu m /(N+1)) .{ }^{3}$ The largest eigenvalue $\chi_{0}$ is unity $\left(\chi_{0}=1\right)$ since a stable stationary solution $\left(\rho_{m}=\right.$ const.) exists. It corresponds to the homogeneous distribution along the chain. Formally this is a consequence of the sum rule (2.1) which expresses the fact that the dynamics can reach any point along the $x$-axis. The non-vanishing eigenvalues are complex and of the form (cf. Appendix A)

$$
\chi_{\nu}^{(\mathrm{p})}=1-(r+l)\left[1-\cos \frac{2 \pi v}{N+1}\right]-\mathrm{i}(r-l) \sin \frac{2 \pi v}{N+1}
$$

with $v=0, \ldots, N$. Their imaginary parts indicate that the relaxation towards the stationary state takes place via temporal oscillations superimposed on an exponential decay, as shown in Fig. 3a. For the long-time dynamics the most relevant eigenvalue besides unity corresponds to the slowest decay $v=1$.

(ii) Absorbing-boundary conditions: $\rho_{0}=\rho_{N+1}=0$. The choice for the boundary cells is determined by the fact that only transitions to these cells are allowed but there are no transitions from the boundary cells. Thus the transfer matrices do not have additional entries besides those on and immediately next to the diagonal, and we have to look for eigenfunctions with the property $\rho_{0}=\rho_{N+1}=0$. In this case the eigenvectors are standing waves of exponentially changing amplitude (cf. Fig. 3b) of the form $(r / l)^{m / 2} \sin (\mathrm{i} \pi \nu m /(N+1))$. The spectrum is real and the non-vanishing eigenvalues are given by

$$
\chi_{\nu}^{(\mathrm{a})}=1-(r+l)+2(l r)^{1 / 2} \cos \frac{\pi v}{N+1}
$$

\footnotetext{
${ }^{3}$ If we take the traveling wave form with a negative exponent the imaginary part of (4.1a) changes sign. For simplicity we consider these cases to be equivalent.
} 
with $v=1,2, \ldots, N$. Here the largest eigenvalue corresponds to $v=1$. It is smaller than unity, reflecting the fact that no non-trivial (i.e., different from the empty state, $\rho_{m}=0$ for all $m$ ) stationary state exists. In this case (Fig. 3b) the density decays exponentially.

The two spectra $\chi_{v}^{(\mathrm{p})}$ and $\chi_{v}^{(\mathrm{a})}$ are not identical, reflecting that the relaxation processes in open and in periodic systems are, in general, different. On the other hand, none of the intracell parameters $\left(s_{i}\right)$ or characteristics along the stable manifold (e.g. $\tilde{l}, \tilde{r}$ ) appears in them. This indicates that the temporal scales in the random-walk picture are independent of the microscopic motion inside cells. In our simple model this independence already holds for the dynamics projected to the spatial $x$ variable (cf. [29] for a heuristic discussion of more general cases).

Since the eigenvalues only depend on the jump probabilities $l$ and $r$, we can follow now how the spectra change when taking the macroscopic limit.

\subsection{Spectra in the macroscopic limit}

One can determine the spectra of the advection-diffusion equation by taking the $\operatorname{limit}^{4} a, \tau \rightarrow 0$ and using conditions (3.14). By this we obtain

$$
\gamma_{k}^{(\mathrm{p})}=\mathrm{i} v k+D k^{2} \equiv \frac{v^{2}}{4 D}+D\left(k+\mathrm{i} \frac{v}{2 D}\right)^{2}
$$

with wave numbers $k=2 \pi v / L, v=0,1, \ldots, N$ for the periodic case, and

$$
\gamma_{k}^{(\mathrm{a})}=\frac{v^{2}}{4 D}+D k^{2}
$$

with $k=\pi v / L, v=1, \ldots, N$ for the absorbing-boundary case. These results show that the macroscopic times are on the order of the diffusion time, $L^{2} / D$ or of the drift time $D / v^{2}$. Although the spectra are different, an interesting relation appears: the transformation $k \rightarrow(k-\mathrm{i} v / D) / 2$ relates $\gamma_{m}^{(\mathrm{p})}$ to $\gamma_{m}^{(\mathrm{a})}$. The complex shift and the difference in the range of available wave numbers ${ }^{5}$ reflects the change of the character of the boundary condition.

\section{Chaos characteristics with meaningful macroscopic limits}

In this section, we investigate which characteristics of the chaotic dynamics possess a meaningful macroscopic limit. They are of special importance since they are the only candidates possibly related to macroscopic transport coefficients. After all the latter must not depend on microscopic details of the dynamics. In classical work (for instance [50]) this independence is attributed to the vast separation of microscopic and macroscopic scales, which also applies in the present setting. This is explicitly demonstrated now by writing the respective quantities in a scaling form with a few scale variables composed of ratios of the microscopic and macroscopic length scales. The macroscopic limit is then expressed as a limit where these scaling variables tend to zero.

\subsection{Decay rates}

An important example of a dynamical characteristics possessing a macroscopic limit is the spectrum of the decay rates, in particular the slowest one. In a system with periodic boundary conditions it describes the relaxation to the steady state, and for open systems amounts to the escape rate.

\footnotetext{
4 A more careful discussion of the limit, and justification of the present approach is given in Section 5.

5 The largest wavelength compatible with absorbing and periodic boundary conditions are $L / 2 \pi$ and $L / \pi$, respectively.
} 


\subsubsection{Scaling form}

We write the eigenvalues $\chi_{\nu}^{(\mathrm{p})}$ of the transfer matrix of the periodic case as

$$
\chi_{v}^{(\mathrm{p})}=1-\tau \frac{2 D}{a^{2}}\left[1-\cos \frac{2 \pi \nu a}{L}\right]-\mathrm{i} \frac{\tau v}{a} \sin \frac{2 \pi \nu a}{L} \equiv 1-\tau \frac{v^{2}}{D} H_{\nu}^{(\mathrm{p})}\left(\frac{a}{l_{v}}, \frac{a}{L}\right) .
$$

Here $H_{v}^{(\mathrm{p})}$ represents a complex-valued bivariate scaling function involving the ratios of the microscopic scale $a$ with the system size $L \equiv a(N+1)$ and the characteristic length scale

$$
l_{v} \equiv \frac{D}{v}
$$

of a biased diffusive system, respectively. For this length the time required to pass it with the drift velocity $v$ is on the same order as the diffusional relaxation time $l_{v}^{2} / D$ (or, equivalently $D / v^{2}$ ) for spatial inhomogeneities of this size. Typically, the three length scales characterizing the system are arranged like

$$
a \ll l_{v} \lesssim L
$$

This condition already implies a large system limit, which we define as $a / l_{v}, a / L \rightarrow 0$.

Similar to the case of periodic boundary conditions, the eigenvalues $\chi_{v}^{(a)}$ of the transfer matrix for the absorbingboundary case can be written as

$$
\chi_{v}^{(\mathrm{a})}=1-\tau \frac{2 D}{a^{2}}\left\{1-\left[1-\left(\frac{v a}{2 D}\right)^{2}\right]^{1 / 2} \cos \frac{\pi v a}{L}\right\} \equiv 1-\tau \frac{v^{2}}{D} H_{v}^{(\mathrm{a})}\left(\frac{a}{l_{v}}, \frac{a}{L}\right),
$$

where $H_{v}^{(a)}$ again is a bivariate scaling function.

\subsubsection{Macroscopic limit}

It directly follows from (5.1) and (5.4) that in the limit $\tau v^{2} / D \ll 1, \tau v / L \ll 1$ the continuous-time decay rates $\gamma_{\nu}$ coincide with those of the advection-diffusion equation, i.e., $\chi_{v}=\exp \left(-\gamma_{\nu} \tau\right)$. In particular, in the periodic case the first non-trivial eigenvalue $\chi_{1}^{(\mathrm{p})}$ of the transfer matrix approaches towards the slowest decay rate of the advection-diffusion dynamics as

$$
\gamma_{1}^{(\mathrm{p})} \equiv-\frac{\log \chi_{1}^{(\mathrm{p})}}{\tau}=D \frac{4 \pi^{2}}{L^{2}}\left[1+\mathcal{O}\left(\frac{a}{l_{v}}\right)^{2}\right]+\mathrm{i} v \frac{2 \pi}{L}\left[1+\mathcal{O}\left(\frac{a}{L}\right)^{2}\right] .
$$

Similarly, the continuous-time escape rate $\kappa$ coincides with the slowest rate $\gamma_{1}^{(a)}$ of the dynamics:

$$
\kappa \equiv-\frac{\log \chi_{1}^{(\mathrm{a})}}{\tau}=\frac{v^{2}}{4 D}\left[1+\mathcal{O}\left(\left(\frac{a}{l_{v}}\right)^{2},\left(\frac{a}{L}\right)^{2}\right)\right]+\frac{D \pi^{2}}{L^{2}}\left[1+\mathcal{O}\left(\left(\frac{a}{l_{v}}\right)^{2},\left(\frac{a}{L}\right)^{2}\right)\right],
$$

where we also dropped terms of order $(\tau v / L)\left(a / l_{v}\right)^{2}$ and $(\tau v / L)(a / L)^{2}$, which are smaller than the indicated higher-order terms by the small factor $\tau v / L$. These formulas show that the leading eigenvalues are related to transport coefficients, but in the general case where $D$ and $v$ are non-zero, these eigenvalues alone do not determine both transport coefficients uniquely.

\subsection{Phase-space contraction rate}

\subsubsection{Scaling form}

Another quantity of interest is the average phase-space contraction rate $\bar{\sigma}$, the average of the negative logarithms of the local Jacobians divided by $\tau$. At the same time, $\bar{\sigma}$ is the negative sum of the average Lyapunov exponents 
$\bar{\sigma} \equiv-\left(\lambda_{1}+\lambda_{2}\right)$. It is interesting to observe that the average positive or negative Lyapunov exponent alone never possesses a meaningful macroscopic limit (cf. Eq. (A.20)). Their sum, however, can survive the limit. Using the results of Appendix A we find for the periodic and open case of our multibaker model

$$
\bar{\sigma}^{(\mathrm{p})} \tau=-\left(\sum_{i} s_{i} \ln \frac{\tilde{s}_{i}}{s_{i}}+l \ln \frac{\tilde{l}}{l}+r \ln \frac{\bar{r}}{r}\right),
$$

and

$$
\bar{\sigma}^{(\mathrm{a})} \tau=-\mathrm{e}^{\kappa \tau}\left(\sum_{i} s_{i} \ln \frac{\tilde{s}_{i}}{s_{i}}+(l r)^{1 / 2} \ln \frac{\tilde{l} \tilde{r}}{l r} \cos \frac{\pi}{N+1}\right),
$$

respectively. The average phase-space contraction rates do depend on the microscopic (inter-cell) parameters: $s_{i}$, $\tilde{s}_{i}, \tilde{l}$ and $\tilde{r}$ are all present in the expression.

Meaningful thermodynamic limits can only exist when we can get rid of the dependence on the microscopic parameters. To that end the global Jacobian $J$ on the strips contributing to transport (cf. Eq. (3.17)) must be the same as the local Jacobians on all the strips staying inside the cell in one time step, i.e.,

$$
\frac{\tilde{s}_{i}}{s_{i}}=J \text { for } i=1, \ldots, k \text {. }
$$

The three classes (a)-(c) introduced in Section 2 obey this requirement.

With Eq. (5.8) we find that in the periodic case

$$
\begin{aligned}
\bar{\sigma}^{(\mathrm{p})} \tau & =-\ln J-\tau\left[\frac{D}{a^{2}}\left(1-\frac{v a}{2 D}\right) \ln \frac{1-\varepsilon v a / 2 D}{1-v a / 2 D}+\frac{D}{a^{2}}\left(1+\frac{v a}{2 D}\right) \ln \frac{1+\varepsilon v a / 2 D}{1+v a / 2 D}\right] \\
& =-\ln J+\tau \frac{v^{2}}{D} S^{(\mathrm{p})}\left(\frac{a}{l_{v}}\right)
\end{aligned}
$$

with $S^{(\mathrm{p})}$ as a single variable scaling function.

Similarly, in the absorbing-boundary case

$$
\bar{\sigma}^{(\mathrm{a})} \tau=-\ln J-\tau \mathrm{e}^{\kappa \tau} \frac{D}{a^{2}}\left[1-\left(\frac{v a}{2 D}\right)^{2}\right]^{1 / 2} \ln \frac{1-(\varepsilon v a / 2 D)^{2}}{1-(v a / 2 D)^{2}} \cos \frac{\pi a}{L}=-\ln J+\tau \frac{v^{2}}{D} S^{(\mathrm{a})}\left(\frac{a}{l_{v}}, \frac{a}{L}\right) .
$$

The scaling function $S^{(\mathrm{a})}$ is now bivariate due to the explicit dependence on $L$. For $J=1$, i.e., in the case where the baker map is one-to-one on its phase space, $\bar{\sigma}^{(a)}$ is an even function of the parameter $\varepsilon$. Consequently, the phase-space contraction rate on the saddle of the absorbing-boundary problem is the same in the thermostated case $\varepsilon=-1$ as in the Hamiltonian case $\varepsilon=1$ :

$$
\bar{\sigma}^{(\mathrm{a})} \tau=0 .
$$

This result can be made plausible by observing that trajectories never escaping the (finite) system take approximately the same number of steps towards and against the bias such that the dynamics is area preserving on the average (cf. discussion at the end of Section 2).

\subsubsection{Macroscopic limit}

Carrying out the macroscopic limit for the phase-space contraction rates, we find in the periodic case that

$$
\bar{\sigma}^{(\mathrm{p})}=-\frac{\ln J}{\tau}+\frac{v^{2}}{D} \frac{(\varepsilon-1)^{2}}{4}\left[1+\mathcal{O}\left(\frac{a}{l_{v}}\right)^{2}\right] \text {. }
$$


In the case of absorbing boundaries on the other hand,

$$
\bar{\sigma}^{(\mathrm{a})}=-\frac{\ln J}{\tau}+\frac{v^{2}}{4 D}\left(\varepsilon^{2}-1\right)\left[1+\mathcal{O}\left(\left(\frac{a}{l_{v}}\right)^{2},\left(\frac{a}{L}\right)^{2}\right)\right] .
$$

Notice that the leading order terms are in both cases proportional to $v^{2} / D$.

\section{Discussion}

For a simple dynamical model of large spatial extension, the multibaker map, we explicitly worked out a hierarchy of coarse-graining processes reminiscent of the reduction of a microscopic dynamics to macroscopic time evolution $[47,48]$. Already the simplest kind of coarse graining (projection on the transport axis) makes the dynamics irreversible and compatible with a kind of random walk. A further coarsening accounting for a separation of microscopic vs. thermodynamically relevant large temporal and spatial scales leads to a continuous-time master equation and an advection-diffusion equation, respectively. The discussion clearly illustrates the relevance of an intermediate, coarse-grained description in terms of Master equations for the description of transport processes (cf. [47,49]). This property is indispensable to obtain a meaningful description on the random walk level. The separation of time and length scales required to end up with macroscopically meaningful equations, expresses that the microscopic parameters ( $a$ and $\tau$ of the multibaker) are negligibly small as compared to the macroscopic scales. They do not affect transport coefficients or particle densities.

We investigated transport in the framework of a thermostated system with periodic boundary conditions, and in the escape-rate formalism. The microscopic dynamics is in both cases given by a well-defined dynamical system generating permanent and transient chaos, respectively. Interestingly, most of the chaos characteristics (including the average Lyapunov exponents, fractal dimensions, entropies) do not have a well-defined macroscopic limit. The only exceptions are the average phase-space contraction rate, i.e., the sum of all Lyapunov exponents $\sum \lambda_{i}$, and the escape rate. They are therefore candidates for being related to transport coefficients and characteristics of thermodynamic steady states. In the thermostated setting the average phase-space contraction rate can indeed coincide with the entropy production, but only for a steady state, where the coarse-grained density is stationary and uniform [34]. When, in the spirit of the escape-rate formalism, the same model is subjected to absorbing-boundary conditions the sum of average Lyapunov exponents vanishes, in spite of a non-zero thermodynamic entropy production due to the explicit time evolution of the connected macroscopic densities. Consequently, the relation between phase-space contraction and the entropy-production rate must not be viewed as a fundamental property of dynamical systems, but can at best apply in certain special cases like uniform stationary states of thermostated systems with absorbing-boundary conditions.

Modeling of transport with all aspects of irreversibility, including entropy production, is consequently a much more complex task than the mere recovering of transport equations. In a general non-stationary situation none of the macroscopically well-defined chaos characteristics can fully account for the entropy production since the latter explicitly depends on the instantaneous density distribution in that case. Moreover, as shown earlier [34,39,43], the expression for the local entropy production corresponding to the continuous-time, large-scale dynamics (i.e., the entropy production in the macroscopic limit) coincides with the one obtained from non-equilibrium thermodynamics [46] including all contributions due to local density differences of the macroscopic state. ${ }^{6}$

\footnotetext{
${ }^{6}$ The entropy production per particle is $\sigma^{(\mathrm{irr})}(x, t)=\left[v-D \partial_{x} \rho(x, t) / \rho(x, t)\right]^{2} / D$, where $\rho(x, t)$ denotes the macroscopic limit of projected density (3.4). It corresponds to the continuous-time, large-scale thermostated dynamics (i.e., $J=1, \varepsilon=-1$ in the present paper). In the periodic case the average phase-space contraction rate (5.11a) turns out to be $\bar{\sigma}=v^{2} / D$, and thus $\sigma^{\text {(irr) }}(x, t)-\bar{\sigma}=-2 v \partial_{x} \rho / \rho+D\left(\partial_{x} \rho / \rho\right)^{2}$, which can take a positive as well as a negative sign. In a spatial average with respect to the density $\rho(x, t)$, however, the first term on the right-hand side vanishes so that the average is strictly positive except in the steady state where $\partial_{x} \rho=0$. In the case of absorbing-boundary conditions $\bar{\sigma} \equiv 0$ (cf.
} 
The discussion of multibaker maps clearly shows that non-standard parameters of the dynamics are essential for the modeling of transport processes. These parameters are the transition probabilities $(l, r$ in the multibaker) between coarse-grained regions and the associated local Jacobians $(\tilde{l} / l, \tilde{r} / r$ in the multibaker). The latter do not influence the transport equations. It is $r$ and $l$, a very uncommon set of parameters from the point of view of dynamical systems, which determine the transport coefficients $v$ and $D$.

Some descriptions of entropic aspects of dynamical-system models of transport emphasize the importance of the SRB measure on the chaotic attractor in the thermostated algorithm $[6,9,12]$, of Takagi-function type distributions of area-preserving models with open boundaries [31,35,37], or of fractal structures of hydrodynamic modes [26,42]. Our results show that none of the usual asymptotic chaos characteristics of the microscopic dynamics appear in the transport coefficients.

Based on these observations, we conclude that it is only the tendency of converging towards a microscopically fractal state which is essential in modeling transport processes. In the spirit of statistical mechanics, coarse graining has to be carried out on a mesoscopic level (on the cells of size $a$ in the multibaker) which is large enough to carry a meaningfully defined density. The coarse-grained distribution therefore settles down to a steady state much earlier than the microscopic motion. The traditional chaos characteristics, which focus only on the asymptotic stationary measure of the microscopic dynamics, are therefore inappropriate for the description of the transport process. Only the presence of microscopic chaos and the resulting Markov property of the coarse-grained dynamics are essential for macroscopic transport-its characteristic numbers are, however, not.

\section{Acknowledgements}

We acknowledge useful discussions with Bob Dorfman, Henk van Beijeren, László Mátyás, Oliver Penrose, and Lamberto Rondoni. The research was supported by the Hungarian Research Foundation (OTKA T032423), the Deutsche Forschungsgemeinschaft through the SFB 237, the ESF Collaborative Research Programme REACTOR, and the Schloessmann foundation of the Max Planck Society.

\section{Appendix A. Evaluating chaotic properties from the transfer matrices}

\section{A.1. Bivariate thermodynamics}

For a complete characterization of invariant chaotic sets of two-dimensional maps a bivariate thermodynamic formalism is especially well suited. Among several versions existing in the literature, we choose one that contains the length scales only. In the most general case the measures are also important but since our multibaker chain is piecewise linear, the natural measure and length scales are proportional, and it is sufficient to consider the length scale statistics. Let $l_{j}^{(n)}\left(\tilde{l}_{j}^{(n)}\right)$ denote the length scales generated by the backward (forward) dynamics along the unstable (stable) direction after $n$ applications of the map. Identical subscripts of $l$ and $\tilde{l}$ indicate that these length scales belong to the same symbol sequence in the backward and forward dynamics. Consider then a weighted sum over all symbols containing products of different powers of $l_{j}^{(n)}$ and $\tilde{l}_{j}^{(n)}$ at a fixed iteration number $n$. Such sums are shown in the thermodynamic theory $[53,54]$ to scale exponentially with $n$. It defines a bivariate thermodynamic function $G\left(\beta_{1}, \beta_{2}\right)$ as

$$
\sum_{j} l_{j}^{(n)^{\beta_{1}}} \tilde{l}_{j}^{(n)^{-\beta_{2}}} \sim \mathrm{e}^{-G\left(\beta_{1}, \beta_{2}\right) n \tau},
$$

(5.11b)), and $\partial_{x} \rho / \rho=\partial_{x} \log \rho \neq 0$ whenever $\rho(x, t)$ is not identically vanishing (see [45] for further details). Consequently, the local entropy production typically differs from the average phase-space contraction rate. 
where $\beta_{1}$ and $\beta_{2}$ are the weighting factors for the length scales along the unstable and stable manifolds, respectively.

A few properties of $G$ can be read off immediately. The topological entropy $K_{0}$ is for instance obtained as $K_{0}=-G(0,0)$. Taking one of the weighting factors to be zero, we recover the free energies $F_{1}$ and $F_{2}$ (the negative of which is also called the topological pressure) along the unstable and stable directions, respectively:

$$
G(\beta, 0)=F_{1}(\beta), \quad G(0,-\beta)=F_{2}(\beta) .
$$

The average Lyapunov exponent $\lambda_{1}$ is obtained as

$$
\lambda_{1}=\left.\frac{\mathrm{d}}{\mathrm{d} \beta} F_{1}(\beta)\right|_{\beta=1} .
$$

The fractal dimensions $d_{0}^{(1)}, d_{0}^{(2)}$ of the invariant set along the unstable and stable directions are

$$
F_{k}\left(\beta=d_{0}^{(k)}\right)=0, \quad k=1,2,
$$

and the escape rate appears as

$$
\kappa=F_{1}(1) .
$$

The free energies contain information on the full spectrum of finite time Lyapunov exponents, Renyi entropies and generalized dimensions, too. For the particular formulas describing how to extract them we refer to the literature $[53,55]$. Finally we note that the phase-space contraction rate $\bar{\sigma} \equiv-\left(\lambda_{1}+\lambda_{2}\right)$ can directly be obtained as a derivative of $G$ :

$$
\bar{\sigma}=\left.\frac{\mathrm{d}}{\mathrm{d} \beta} G(1-\beta,-\beta)\right|_{\beta=0} .
$$

For systems with Markov partitions the quantity $\exp (-G \tau)$ appears as the leading eigenvalue of a generalized transition matrix. This matrix has the same structure as the traditional transition matrix just the entries are the same as the length scales at level $n=1$ raised to powers $\beta_{1}, \beta_{2}$. Thus we have the generalized transition matrix $\overline{\mathrm{T}}\left(\beta_{1}, \beta_{2}\right)$ for the baker chain also in a tridiagonal from with non-vanishing elements

$$
\begin{aligned}
& \bar{T}_{m, m-1}\left(\beta_{1}, \beta_{2}\right)=l^{\beta_{1}} \tilde{l}^{-\beta_{2}} \equiv \mathrm{l}, \\
& \bar{T}_{m, m}\left(\beta_{1}, \beta_{2}\right)=\sum_{i} s_{i}^{\beta_{1}} \tilde{s}_{i}^{-\beta_{2}} \equiv \mathrm{s}, \\
& \bar{T}_{m, m+1}\left(\beta_{1}, \beta_{2}\right)=r^{\beta_{1}} \tilde{r}^{-\beta_{2}} \equiv \mathrm{r} .
\end{aligned}
$$

\section{A.2. The spectrum of tridiagonal matrices}

Because tridiagonal matrices appear in several forms in our problem, let us consider the eigenvalue problem of a general $N \times N$ matrix with diagonal elements $\mathrm{S}$ and off diagonal elements $\mathrm{r}$ and $\mathrm{l}$. The eigenvalue equation for the non-vanishing eigenvalues $\chi$ of $\overline{\mathrm{T}}$ is

$$
\mathrm{r} u_{m-1}+\mathrm{s} u_{m}+\mathrm{l} u_{m+1}=\chi u_{m} .
$$

In the case of constant elements exponential solutions are expected for the eigenvectors $u_{m}, m=1, \ldots, N$. 
Let us first assume a traveling wave form for the eigenvectors:

$$
u_{m}=\mathrm{e}^{\mathrm{i} m \theta} .
$$

Substitution of this into (A.8) yields a complex set of eigenvalues

$$
\chi=s+(r+I) \cos \theta-i(r-I) \sin \theta .
$$

These are consistent with periodicity required by the condition $u_{1}=u_{N+1}$ provided that $\theta_{v}=2 \pi v /(N+1)$ with $v=0,1, \ldots, N$. Thus the spectrum in the presence of periodic boundary conditions reads as

$$
\chi_{v}^{(\mathrm{p})}=\mathrm{s}+(\mathrm{r}+\mathrm{l}) \cos \frac{2 \pi v}{N+1}-\mathrm{i}(\mathrm{r}-\mathrm{l}) \sin \frac{2 \pi v}{N+1}
$$

with $v=0,1, \ldots, N$. Now the largest eigenvalue

$$
\chi_{0}^{(p)}=s+(r+l)
$$

is the only real element of the spectrum (for $r \neq \mathrm{I}$ ) and is independent of the system size.

A different type of solutions is found by looking for real solutions in the form

$$
u_{m}=\mathrm{e}^{m \alpha} \sin (m \theta) \text {. }
$$

A direct substitution into (A.8) then specifies the exponent $\alpha$ as

$$
\alpha=\frac{1}{2} \ln \frac{r}{i}
$$

and yields for the eigenvalue

$$
\chi=\mathrm{s}+2 \sqrt{\mathrm{rl}} \cos \theta .
$$

This solution corresponds to a standing wave with an exponentially increasing amplitude in space and is only compatible with an absorbing-boundary condition. By requiring free ends with $u_{0}=u_{N+1}=0$ we find that $\theta$ can only take on values $\theta_{v}=v \pi /(N+1), v=1, \ldots, N$. Thus, the entire spectrum belonging to absorbing-boundary conditions is (apart from degenerate zero eigenvalues)

$$
\chi_{v}^{(\mathrm{a})}=\mathrm{s}+2 \sqrt{\mathrm{rl}} \cos \frac{v \pi}{N+1}, \quad v=1,2, \ldots, N .
$$

The largest eigenvalue is that of $v=1$. Note that the size dependence is present in all the elements but a large system limit $N \rightarrow \infty$ exists. Note that the two spectra are qualitatively different, the largest eigenvalues coincide not even in the large $N$ limit (cf. Fig. 4).

\section{A.3. Characterizing the invariant sets}

Substituting the non-vanishing matrix elements of $\mathrm{T}\left(\beta_{1}, \beta_{2}\right)$ for the periodic and absorbing-boundary conditions into the respective largest eigenvalues yields two different bivariate potentials $G^{(\mathrm{p})}$ and $G^{(\mathrm{a})}$, viz.

$$
\begin{aligned}
\mathrm{e}^{-G^{(\mathrm{p})}\left(\beta_{1}, \beta_{2}\right) \tau} & =\sum_{i} s_{i}^{\beta_{1}} \tilde{s}_{i}^{-\beta_{2}}+l^{\beta_{1}} \tilde{l}^{-\beta_{2}}+r^{\beta_{1}} \tilde{r}^{-\beta_{2}}, \\
\mathrm{e}^{-G^{(\mathrm{a})}\left(\beta_{1}, \beta_{2}\right) \tau} & =\sum_{i} s_{i}^{\beta_{1}} \tilde{s}_{i}^{-\beta_{2}}+2(l r)^{\beta_{1} / 2}(\tilde{l} \tilde{r})^{-\beta_{2} / 2} \cos \frac{\pi}{N+1} .
\end{aligned}
$$



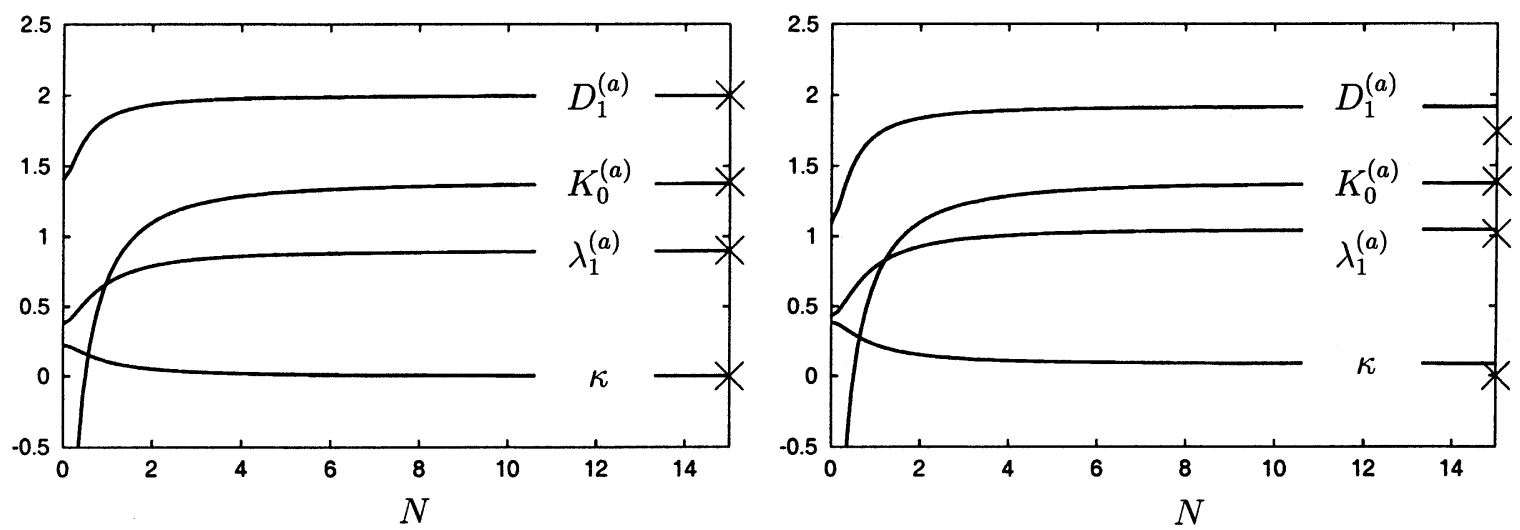

Fig. 4. The $N$-dependence of different quantities characterizing the chaotic set for a system with absorbing-boundary conditions. The crosses at the right border give the corresponding values for periodic boundary conditions (which do not depend on $N$ ). The symbols are explained in the text, and time is measured in units of $\tau$ (parameters-left: $k=2, s_{1}=0.675, s_{2}=0.225, r=0.052, l=0.048$, as in Fig. 3; right: $k=2$, $\left.s_{1}=0.6, s_{2}=0.2, r=0.18, l=0.02\right)$.

The corresponding free energies also depend on the boundary condition, and therefore, the spectra of local Lyapunov exponents will typically be different for the open and periodic cases. Here, we just give some important chaos characteristics explicitly. The topological entropies are obtained as

$$
\begin{aligned}
& K_{0}^{(\mathrm{p})} \tau=\ln (k+2), \\
& K_{0}^{(\mathrm{a})} \tau=\ln \left[k+2 \cos \frac{\pi}{N+1}\right],
\end{aligned}
$$

which shows that the symbolic dynamics is never complete in a finite, open system. ${ }^{7}$ Note also that even if the single-cell dynamics is non-chaotic (i.e., $k=0$ or $k=1$ ) the spatially-extended system, where $N \gg 1$, is always chaotic.

For the escape rate we find,

$$
\kappa \tau=-\ln \left[(1-l-r)+2 \sqrt{\operatorname{lr}} \cos \frac{\pi}{N+1}\right] .
$$

It is independent of the microscopic quantities $s_{i}$ but contains the jump probabilities $l, r$ related to the transport coefficients. $\beta_{1}=1, \beta_{2}=0$ is the only "temperature" setting in the thermodynamic formalism where this can happen.

The positive Lyapunov exponents for the respective boundary conditions are

$$
\begin{aligned}
& \lambda_{1}^{(\mathrm{p})} \tau=-\sum_{i} s_{i} \ln s_{i}-l \ln l-r \ln r \\
& \lambda_{1}^{(\mathrm{a})} \tau=\mathrm{e}^{\kappa \tau}\left[-\sum_{i} s_{i} \ln s_{i}-(l r)^{1 / 2} \ln (l r) \cos \frac{\pi}{N+1}\right] .
\end{aligned}
$$

\footnotetext{
7 The escape of particle trajectories characterized by certain symbol sequences introduces pruning in the symbolic dynamics. Since there is less and less escape for $N \rightarrow \infty$, however, $K_{0}^{(\mathrm{a})}$ and $K_{0}^{(\mathrm{p})}$ become identical in the large $N$ limit (cf. Fig. 4).
} 
These quantities, for instance, do not possess a macroscopic limit in the spirit of Section 3.5 because the dependence of the terms $l \ln l$ and $r \ln r$ on the microscopic time and space units $\tau$ and $a$ is not removed in the limit (cf. Eq. (3.16)).

The metric entropies are

$$
K_{1}^{(\mathrm{p})}=\lambda_{1}^{(\mathrm{p})}, \quad K_{1}^{(\mathrm{a})}=\lambda_{1}^{(\mathrm{a})}-\kappa,
$$

and from the second derivative of $G$ (A.6) we obtain for the phase-space contraction rates

$$
\begin{aligned}
& \bar{\sigma}^{(\mathrm{p})} \tau=-\left(\sum_{i} s_{i} \ln \frac{\tilde{s}_{i}}{s_{i}}+l \ln \frac{\tilde{l}}{l}+r \ln \frac{\tilde{r}}{r}\right), \\
& \bar{\sigma}^{(\mathrm{a})} \tau=-\mathrm{e}^{\kappa \tau}\left[\sum_{i} s_{i} \ln \frac{\tilde{s}_{i}}{s_{i}}+(l r)^{1 / 2} \ln \frac{\tilde{l} \tilde{r}}{l r} \cos \frac{\pi}{N+1}\right] .
\end{aligned}
$$

The information dimension of the chaotic set's unstable manifolds can be written as

$$
\begin{aligned}
& D_{1}^{(\mathrm{p})}=1+d_{1}^{(2, p)}=2+\frac{\sigma^{(\mathrm{p})}}{\left|\lambda_{2}^{(\mathrm{p})}\right|}, \\
& D_{1}^{(\mathrm{a})}=1+d_{1}^{(2, a)}=2+\frac{\sigma^{(\mathrm{a})}-\kappa}{\left|\lambda_{2}^{(\mathrm{a})}\right|} .
\end{aligned}
$$

The denominator contains in both cases the Lyapunov exponent characterizing the stable manifold. Since the Lyapunov exponent does not possess a macroscopic limit, neither does the information dimension. It is remarkable, however, that the combination $\left(2-D_{1}\right) \lambda_{2}$, which is the difference of the phase-space contraction and escape rate, is macroscopically well defined for both boundary conditions considered (note that $\kappa \tau=0$ for periodic boundary conditions).

\section{References}

[1] E. Hopf, Ergodentheorie, Springer, Berlin, 1937.

[2] D.J. Evans, G.P. Morriss, Statistical Mechanics of Nonequilibrium Liquids, Academic Press, London, 1990; W.G. Hoover, Computational Statistical Mechanics, Elsevier, Amsterdam, 1991.

[3] J.R. Dorfman, From Molecular Chaos to Dynamical Chaos, Cambridge University Press, Cambridge, 1999.

[4] P. Gaspard, Chaos, Scattering and Statistical Mechanics, Cambridge University Press, Cambridge, 1999.

[5] W.G. Hoover, Time Reversibility, Computer Simulation, and Chaos, World Scientific, Singapore, 1999.

[6] D. Ruelle, J. Statist. Phys. 95 (1999) 392.

[7] D. Szász (Ed.), Hard Ball Systems and the Lorentz Gas, Springer Encyclopædia of Mathematical Sciences, vol. 101, Springer, Berlin, 2000.

[8] J. Vollmer, Phys. Rep. 372 (2002) 131-267.

[9] G. Gallavotti, E.G.D. Cohen, Phys. Rev. Lett. 74 (1995) 2694;

G. Gallavotti, E.G.D. Cohen, J. Statist. Phys. 80 (1995) 931;

G. Gallavotti, Chaos 8 (1998) 384.

[10] P. Gaspard, G. Nicolis, Phys. Rev. Lett. 65 (1990) 1693.

[11] W.N. Vance, Phys. Rev. Lett. 69 (1992) 1356.

[12] N.I. Chernov, G.L. Eyink, J.L. Lebowitz, Ya.G. Sinai, Phys. Rev. Lett. 70 (1993) 2209; N.I. Chernov, G.L. Eyink, J.L. Lebowitz, Ya.G. Sinai, Commun. Math. Phys. 154 (1993) 569.

[13] R. Klages, J.R. Dorfman, Phys. Rev. Lett. 74 (1995) 387.

[14] G. Radons, Phys. Rev. Lett. 77 (1996) 4748.

[15] J.R. Dorfman, H. van Beijeren, Physica A 240 (1997) 12.

[16] E.G.D. Cohen, L. Rondoni, Chaos 8 (1998) 357.

[17] W.G. Hoover, A. Posch, Chaos 8 (1998) 366. 
[18] G.P. Morriss, L. Rondoni, J. Statist. Phys. 75 (1994) 553; G.P. Morriss, L. Rondoni, Physica A 233 (1996) 767; L. Rondoni, G.P. Morriss, Phys. Rev. E 53 (1996) 2143; C.P. Dettmann, G.P. Morriss, L. Rondoni, Chaos, Solitons and Fractals 8 (1997) 783; G.P. Morriss, C.P. Dettmann, Chaos 8 (1998) 321.

[19] S. Nielsen, R. Kapral, J. Chem. Phys. 109 (1998) 6460.

[20] D. Alonso, R. Artuso, G. Casati, I. Guarneri, Phys. Rev. Lett. 82 (1999) 1859.

[21] J.-P. Eckmann, C.-A. Pillet, L. Rey-Bellet, Commun. Math. Phys. 201 (1999) 657; J.-P. Eckmann, M. Hairer, Commun. Math. Phys. 212 (2000) 105.

[22] C.P. Dettmann, in: D. Szász (Ed.), Hard Ball Systems and the Lorentz Gas, Springer Encyclopædia of Mathematical Sciences, vol. 101, Springer, Berlin, 2000, pp. 315-366.

[23] D.J. Evans, E.G.D. Cohen, D.J. Searles, F. Bonetto, J. Statist. Phys. 101 (2000) 17.

[24] K. Rateischak, R. Klages, W.G. Hoover, J. Statist. Phys. 101 (2000) 61.

[25] I. Claus, P. Gaspard, J. Statist. Phys. 101 (2000) 161.

[26] P. Gaspard, I. Claus, T. Gilbert, J.R. Dorfman, Phys. Rev. Lett. 86 (2001) 1506; T. Gilbert, J.R. Dorfman, P. Gaspard, Nonlinearity 14 (2001) 339.

[27] P. Gaspard, Adv. Chem. Phys. 122 (2002) 109.

[28] P. Gaspard, I. Claus, Philos. Trans. R. Soc. A 360 (2002) 303.

[29] D.J. Evans, L. Rondoni, J. Statist. Phys. 109 (2002) 895.

[30] P. Gaspard, J. Statist. Phys. 68 (1992) 673.

[31] S. Tasaki, P. Gaspard, J. Statist. Phys. 81 (1995) 935.

[32] T. Tél, J. Vollmer, W. Breymann, Europhys. Lett. 35 (1996) 659.

[33] W. Breymann, T. Tél, J. Vollmer, Phys. Rev. Lett. 77 (1996) 2945.

[34] J. Vollmer, T. Tél, W. Breymann, Phys. Rev. Lett. 79 (1997) 2759; J. Vollmer, T. Tél, W. Breymann, Phys. Rev. E 58 (1998) 1672; W. Breymann, T. Tél, J. Vollmer, Chaos 8 (1998) 396.

[35] P. Gaspard, J. Statist. Phys. 88 (1997) 1215.

[36] P. Gaspard, R. Klages, Chaos 8 (1998) 409.

[37] S. Tasaki, T. Gilbert, J.R. Dorfman, Chaos 8 (1998) 424; T. Gilbert, J.R. Dorfman, J. Statist. Phys. 96 (1999) 225.

[38] S. Tasaki, P. Gaspard, Theoret. Chem. Acc. 102 (1999) 385; S. Tasaki, P. Gaspard, J. Statist. Phys. 101 (2000) 125.

[39] L. Mátyás, T. Tél, J. Vollmer, Phys. Rev. E 61 (2000) R3295; L. Mátyás, T. Tél, Phys. Rev. E 62 (2000) 349; J. Vollmer, T. Tél, L. Mátyás, J. Statist. Phys. 101 (2000) 79.

[40] L. Rondoni, E.G.D. Cohen, Nonlinearity 13 (2000) 1905; E.G.D. Cohen, L. Rondoni, Physica A 306 (2002) 117; L. Rondoni, E.G.D. Cohen, Physica D 168-169 (2002) 341.

[41] T. Tél, J. Vollmer, in: D. Szász (Ed.), Hard Ball Systems and the Lorentz Gas, Springer Encyclopædia of Mathematical Sciences, vol. 101, Springer, Berlin, 2000, pp. 367-420.

[42] T. Gilbert, J.R. Dorfman, P. Gaspard, Phys. Rev. Lett. 85 (2000) 1606.

[43] T. Tél, J. Vollmer, L. Mátyás, Europhys. Lett. 53 (2001) 458.

[44] S. Tasaki, Adv. Chem. Phys. 122 (2002) 77.

[45] J. Vollmer, L. Mátyás, T. Tél, J. Statist. Phys. 109 (2002) 875. nlin.CD/0112021.

[46] S.R. de Groot, P. Mazur, Nonequilibrium Thermodynamics, Elsevier, Amsterdam, 1962.

[47] N.G. van Kampen, in: E.G.D. Cohen (Ed.), Fundamental Problems in Statistical Physics, North-Holland, Amsterdam, 1962, pp. 173-202.

[48] H. Grad, in: M. Bunge (Ed.), Studies in the Foundations, Methodology and Philosophy of Science, vol. I, Springer, Berlin, 1967.

[49] O. Penrose, Foundations of Statistical Mechanics: A Deductive Treatment, Pergamon Press, Oxford, 1970;

O. Penrose, Rep. Prog. Phys. 42 (1979) 1937.

[50] N.G. van Kampen, J. Statist. Phys. 46 (1987) 709.

[51] J.P. Eckmann, D. Ruelle, Rev. Mod. Phys. 57 (1985) 617.

[52] M.J. Feigenbaum, M.H. Jensen, I. Procaccia, Phys. Rev. Lett. 57 (1986) 1503; T. Bohr, M.H. Jensen, Phys. Rev. A 36 (1987) 4904.

[53] C. Beck, F. Schlögl, Thermodynamics of Chaotic Systems, Cambridge University Press, Cambridge, 1993.

[54] S.M. Ulam, A Collection of Mathematical Problems, vol. 8, Interscience, New York, 1960, p. 73; T.Y. Li, Approximation Theory 17 (1976) 177; Z. Kovács, T. Tél, Phys. Rev. A 40 (1989) 4641.

[55] Z. Kovács, T. Tél, Phys. Rev. Lett. 64 (1990) 1617.

[56] W. Feller, An Introduction to Probability Theory and its Applications, Wiley, New York, 1978.

[57] C. Gardiner, Handbook for Stochastic Processes, Springer, Berlin, 1983. 